\title{
Hot tub lung mimicking classic acute and chronic hypersensitivity pneumonitis: Two case reports
}

\author{
Geetika Verma MD FRCPC ${ }^{1}$, Frances Jamieson MD FRCPC ${ }^{2}$, Pamela Chedore $\mathrm{MLT}^{3}$, \\ David Hwang BSC MD PhD FRCPC ${ }^{4}$, Scott Boerner MD FRCPC ${ }^{4,5}$, William R Geddie MD FRCPC ${ }^{4}$, \\ Kenneth R Chapman MD FRCPC ${ }^{1}$, Theodore K Marras MD FRCPC MSc ${ }^{1}$
}

G Verma, F Jamieson, P Chedore, et al. Hot tub lung mimicking classic acute and chronic hypersensitivity pneumonitis: Two case reports. Can Respir J 2007;14(6):354-356.

Pulmonary disease in otherwise healthy patients can occur by secondary exposure to nontuberculous mycobacteria from hot tubs. The pathology of hot tub lung may be related to an infection, a hypersensitivity reaction or both. Previous reports of hot tub lung have highlighted distinct pathological features that have distinguished this entity from classic hypersensitivity pneumonitis. Two cases of hot tub lung in Ontario, which presented at very different time points in their disease course, are reported; one patient presented more fulminantly with a clinical picture resembling subacute hypersensitivity pneumonitis, and the other presented with chronic disease. Both cases exhibited clinical, radiological and pathological findings closely mimicking classic subacute and chronic hypersensitivity pneumonitis.

Key Words: Hot tub; Hypersensitivity pneumonitis; Nontuberculous mycobacteria

$\mathrm{N}$ ontuberculous mycobacteria (NTM) are environmental pathogens that occur in both natural and municipal water sources, and proliferate in warm temperatures (1). While infection usually occurs in patients with underlying lung disease or impaired immunity, exposure to aerosolized NTM from hot tubs has been described to cause an NTM infection, a hypersensitivity reaction or both in otherwise healthy individuals $(2,3)$.

We present two cases of probable hot tub lung with different presentations. The first had an indolent presentation similar to chronic hypersensitivity pneumonitis (HP). His problem remained undiagnosed for approximately 10 years. The second individual presented with more acute symptoms similar to acute HP, leading to prompt investigations that provided clues to NTM exposure. In both cases, exposure through hot tub use was determined in retrospect, given the radiographic and pathological findings.

\section{CASE PRESENTATION}

Case 1

A 53-year-old man with a six-month history of cough presented with purulent sputum and increased dyspnea. His medical history was unremarkable.

He had experienced similar respiratory symptoms 10 years previously, when pulmonary function tests demonstrated

\section{Alvéolite des utilisateurs de spas prenant l'aspect d'une pneumopathie d'hypersensibilité : Deux rapports de cas}

\begin{abstract}
Chez des sujets par ailleurs en bonne santé, la maladie pulmonaire peut être consécutive à une exposition à des mycobactéries non tuberculeuses présentes dans l'eau des spas. La pathologie de l'alvéolite des utilisateurs de spas (ou hot tub lung) peut découler d'une infection, d'une hypersensibilité ou des deux. De précédents rapports sur ce type d'alvéolite ont fait ressortir les différences entre cette affection et la pneumopathie d'hypersensibilité classique. Deux cas d'alvéolite des utilisateurs de spas ont été recensés en Ontario; les deux patients ont consulté à un stade différent de la maladie. L'un des cas s'est révélé plus fulminant, et le tableau clinique ressemblait à celui d'une pneumopathie d'hypersensibilité subaiguë et dans l'autre cas, la pneumopathie était chronique. Dans les deux cas, les signes cliniques, radiologiques et pathologiques ressemblaient étroitement à ceux de la pneumopathie d'hypersensibilité subaiguë et chronique.
\end{abstract}

obstruction without reversibility. He was treated for possible asthma with inhaled medications and prednisone, showing subsequent improvement in symptoms. A chest radiograph was unremarkable, and a computed tomography (CT) scan of the thorax was performed given the patient's steroid dependence. The findings were consistent with bronchiolitis obliterans. In subsequent years, he had inconsistent follow-up, but continued to use inhaled corticosteroids regularly.

Pulmonary function testing at his current presentation showed severe obstruction (forced expiratory volume in $1 \mathrm{~s}$ $20 \%$ of predicted), mild hyperinflation, gas trapping at residual volume and normal diffusion. His CT scan demonstrated mosaic attenuation, consistent with air trapping, and diffuse micronodularity (Figure 1).

A surgical lung biopsy demonstrated cellular and constrictive bronchiolitis, with predominantly lymphocytic inflammation and numerous poorly formed granulomas, suggestive of HP. Simultaneously, collected bronchoalveolar lavage (BAL) was negative for acid-fast bacilli (AFB) but culture positive for Mycobacterium avium complex (MAC) (Figure 2).

The patient had no significant occupational exposures. He did not keep birds. Ten years previously, coincident with the onset of his initial symptoms, he moved into a new home, where he installed a hot tub in a poorly ventilated room that he used regularly.

${ }^{1}$ Department of Medicine, Division of Respirology, University Health Network, University of Toronto; ${ }^{2}$ Clinical and Environmental Microbiology,

Public Health Laboratories Branch, Ontario Ministry of Health and Long-Term Care; ${ }^{3}$ Tuberculosis and Mycobacteriology Laboratory, Public

Health Laboratories Branch, Ontario Ministry of Health and Long-Term Care; ${ }^{4}$ Department of Pathology; ${ }^{5}$ Department of Laboratory

Medicine and Pathobiology, University Health Network, University of Toronto, Toronto, Ontario

Correspondence: Dr Theodore K Marras, Toronto Western Hospital, 7E-452, 399 Bathurst Street, Toronto, Ontario M5T 2 S8.

Telephone 416-603-5767, fax 416-603-5375, e-mail ted.marras@uhn.on.ca 


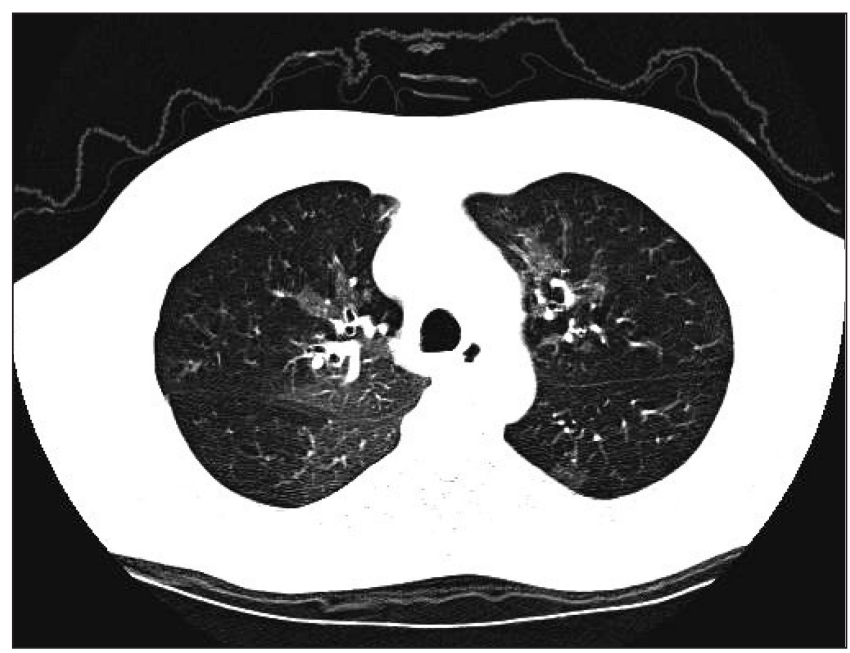

Figure 1) Case 1: Computed tomography of the chest with axial slice demonstrating mosaic attenuation in keeping with small airway obstruction

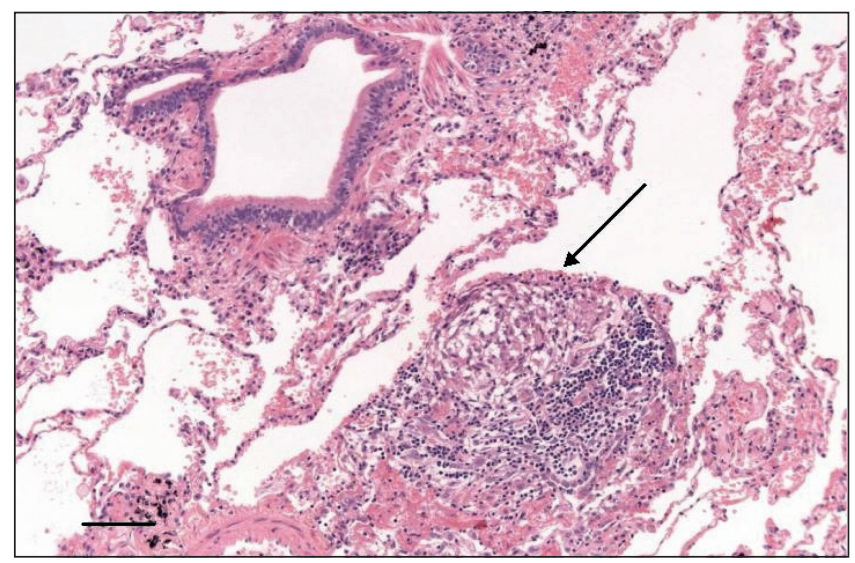

Figure 2) Case 1: Lung biopsy showing patchy bronchiolocentric chronic inflammatory infiltrates and a poorly formed granuloma (arrow). Original magnification $\times 100$; Scale bar $0.2 \mathrm{~mm}$

Based on the constellation of findings, he was advised to discontinue hot tub use during his initial presentation. Within 10 months, he improved using inhaled medications and hot tub avoidance alone, but repeat sputum culture remained positive for MAC and his exercise tolerance was impaired. He started antimycobacterial therapy (1000 mg of clarithromycin, $1200 \mathrm{mg}$ of ethambutol and $600 \mathrm{mg}$ of rifampin every Monday, Wednesday and Friday) and prednisone (40 mg daily for two weeks, then $20 \mathrm{mg}$ daily for six weeks). He had no obvious initial response to prednisone, but within two months of starting antimycobacterial therapy, his forced expiratory volume in $1 \mathrm{~s}$ improved to $2 \mathrm{~L}$ ( $55 \%$ of predicted). His sputum culture was negative for MAC after 12 weeks, by which time he was asymptomatic. He continued anti-NTM treatment for eight months.

Home environmental sampling of biofilm from the hot tub jets, filter and drain was performed. Two of the three swabs from the jets were positive for AFB, and all three grew MAC. Both swabs from the filter were positive for AFB and grew numerous MAC. Similarly, the drain swab was positive for AFB and grew MAC. Molecular typing of the isolates was not performed, because the clinical isolate was not available at the time of environmental sampling.
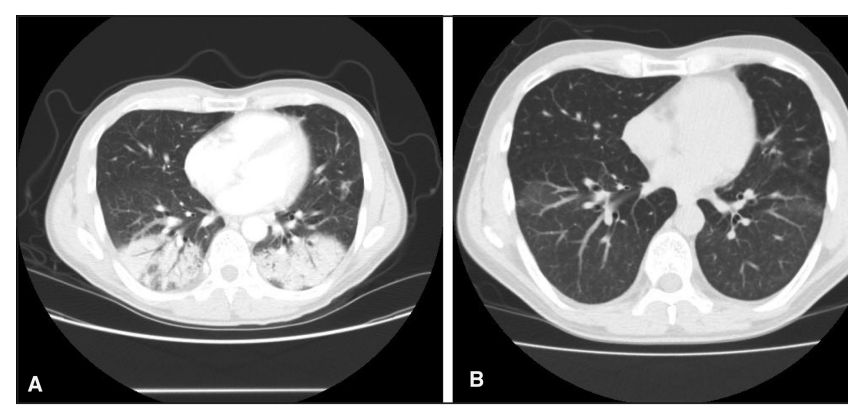

Figure 3) Case 2: Computed tomography scans of the chest at presentation (A) and after two months (B). The striking bilateral lower lobe consolidation in A improved, though areas of ground glass attenuation persisted

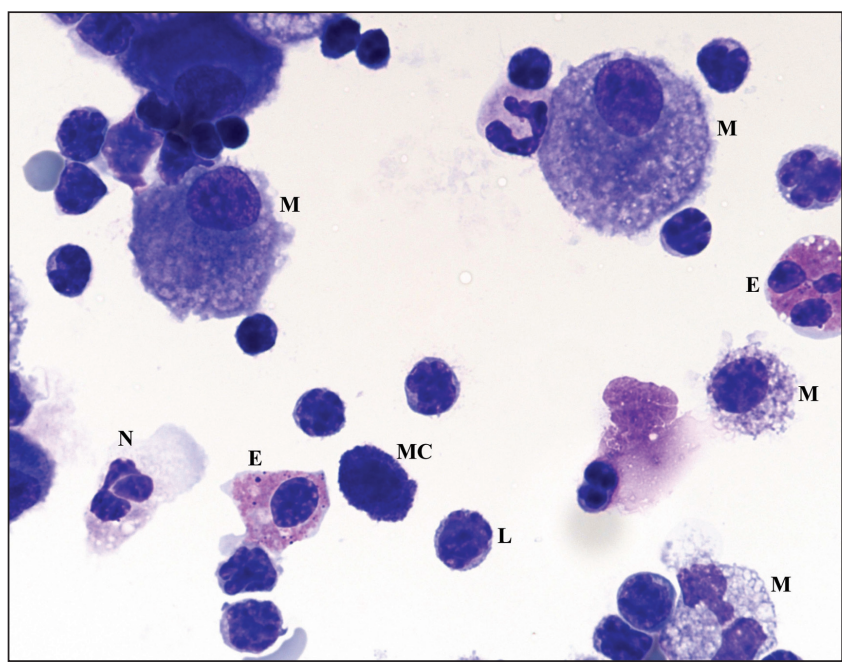

Figure 4) Case 2: Bronchoalveolar lavage fluid cytospin preparation. This field shows pulmonary alveolar macrophages (M), neutrophils $(N)$, eosinophils (E), numerous lymphocytes (L) and a mast cell (MC). Original magnification $\times 100$

Case 2

A 49-year-old man was referred for a two-month history of fever, weight loss, dyspnea and productive cough. He was treated with three courses of antibiotics without improvement. In the initial encounter, a relevant exposure history was not elicited.

Chest $\mathrm{x}$-ray showed bilateral lower lobe opacities, and CT revealed bilateral apical interlobular septal thickening, as well as striking ground glass opacification and consolidation, in the lower lobes (Figures $3 \mathrm{~A}$ and $3 \mathrm{~B}$ ).

BAL demonstrated marked lymphocytosis (70\%) with a CD4 to CD8 ratio of 1:5, as well as numerous mast cells and eosinophils, all suggestive of HP. BAL cultures and AFB testing were negative (Figure 4).

On further questioning, the patient admitted to daily hot tub use. He was told to discontinue hot tub use and was prescribed prednisone $(50 \mathrm{mg} /$ day $)$. In the interim, sputum cultures collected by his family physician when he first developed symptoms grew Mycobacterium fortuitum. Given his persistent symptoms, he was started on clarithromycin and levofloxacin. Two months after presentation and six weeks after initiating treatment, his CT scan showed complete resolution of the consolidation, with some residual ground glass opacification. Clinically, he had improved dramatically, with resolution of his cough and return to his baseline weight. The patient elected to stop prednisone and antibiotics after three weeks of therapy. 
Two months later (six months from presentation), he returned with fatigue and exertional dyspnea. Repeat bronchoscopy demonstrated a decreased lymphocytosis of $15 \%$, with a CD4 to CD8 ratio of 2:1 and negative cultures. Repeat CT imaging showed some improvement but also interval development of new areas of ground glass. He admitted resuming hot tub use. He was again advised to avoid the hot tub and to restart prednisone. After three months of continued use of prednisone, his symptoms improved. Antimycobacterial therapy was never resumed.

Cultures from his hot tub drain and jets, as well as his showerhead and drain, were examined. A swab from one of the two hot tub jets was positive for AFB, and swabs from the showerhead were negative for AFB; both grew Mycobacterium gordonae. The hot tub drain and shower drain swabs were smear positive, with cultures demonstrating $M$ fortuitum. Further genotyping of the $M$ fortuitum isolated from the drains and the patient's sputum was not performed, because the original sputum isolates were no longer available when environmental sampling was performed.

\section{DISCUSSION}

There has been debate whether hot tub lung is predominantly a hypersensitivity reaction versus an infection. We have presented two cases of probable hot tub lung that bore similarity to opposite ends of the HP 'acuity' spectrum; the first was akin to chronic HP and the second was more consistent with subacute HP.

HP usually presents with a flu-like syndrome and cough in more acute forms, and with exertional dyspnea, fatigue and productive cough in chronic disease. High-resolution CT findings include ground glass opacification and/or consolidation in the acute setting, plus centrilobular nodularity. In chronic forms, reticular changes and fibrosis may predominate (4). Mosaic attenuation due to small airway obstruction may occur at any stage (5). In HP, the BAL findings are classically described as demonstrating marked lymphocytosis with a low CD4 to CD8 ratio (6).

MAC infection is typically characterized radiographically by bronchiectasis and nodular infiltrates, plus or minus cavitation, and pathologically by necrotizing granulomatous inflammation with demonstration of the organism in most cases (7). BAL findings in MAC lung infection have consistently included neutrophilia and lymphocytosis $(8,9)$, but the CD4 to CD8 ratio has been reported variably as elevated (8) or normal (9).

Marras et al (2) distinguished NTM-related hypersensitivity reactions from classic HP (2). In their review, patients with NTM-related HP had elevated BAL CD4 to CD8 ratios, and well-formed granulomas on biopsy, both atypical for classic HP. Hanak et al (3) reviewed 21 cases of hot tub lung, which they concluded resembled HP more closely than M avium - intracellulare infection. High-resolution CT scans demonstrated centrilobular micronodules, ground glass opacification and air trapping in those patients in whom expiratory views were performed. Similar to a review by Marras et al (2), BAL findings included marked lymphocytosis and elevated CD4 to CD8 ratios, and lung biopsy specimens consisted of well-formed granulomas and chronic inflammation (3). Both of our patients differed from these descriptions. In case 1, the biopsy contained loosely formed, poorly circumscribed granulomas without demonstration of mycobacterial organisms, which is seen in classic HP (7). In case 2, the low BAL CD4 to CD8 ratio differed from traditional descriptions of hot tub lung. In both cases, radiographic findings also resembled classic HP. We believe that the differences between our cases and previously reported cases $(1-3,7)$ of hot tub lung support the notion that hot tub lung is heterogeneous in its presentation and may be strikingly similar to classic HP.

Although we believe that the diagnosis of hot tub lung is justified in both cases, some features of case 2 warrant additional discussion. The radiographic consolidation, low BAL CD4 to CD8 ratio and response to prednisone raise the possibility of cryptogenic organizing pneumonia. We believe that the relationship between the repeated exposure to the hot tub and the patient's symptoms, as well as the recovery of NTM from both the patient's sputum and the hot tub and shower drains, strongly favour hot tub lung. Furthermore, the BAL findings of mast cells and marked lymphocytosis of $70 \%$ are uncommon in cryptogenic organizing pneumonia (10). Secondly, the causative organism was $M$ fortuitum, an organism better known for causing cutaneous infections. Although most cases of hot tub lung described have been due to MAC, Mangione et al (1) described four patients with hot tub lung, one of whom had both sputum and hot tub sample cultures positive for M fortuitum (1).

Our cases illustrate that hot tub lung can present very similarly to both acute and chronic HP. Previous reports $(1-3,7)$ of HP-like syndromes due to NTM exposure have not demonstrated pathological findings in keeping with 'classic' HP. Although we believe that the distinction between NTM infection and hypersensitivity is somewhat semantic, this syndrome appears to bear remarkable pathophysiological and histological similarities to classic HP.

ACKNOWLEDGEMENTS: We wish to acknowledge the clinical contributions of Dr TK Waddell, Division of Thoracic Surgery, University Health Network, Toronto, Ontario.

\section{REFERENCES}

1. Mangione EJ, Huitt G, Lenaway D, et al. Nontuberculosis myobacterial disease following hot tub exposure. Emerg Infect Dis 2001;7:1039-42.

2. Marras TK, Wallace RJ Jr, Koth LL, Stulberg MS, Cowl CT, Daley CL. Hypersensitivity pneumonitis reaction to Mycobacterium avium in household water. Chest 2005;127:664-71.

3. Hanak V, Kalra S, Aksamit TR, Hartman TE, Tazelaar HD, Ryu JH. Hot tub lung: Presenting features and clinical course of 21 patients. Respir Med 2006;100:610-5.

4. Selman M. Hypersensitivity pneumonitis. In: Schwarz MI, King TE, eds. Interstitial Lung Disease, 4th edn. Hamilton: BC Decker, 2003:452-84.

5. Hansell DM, Moskovic E. High-resolution computed tomography in extrinsic allergic alveolitis. Clin Radiol 1991;43:8-12.

6. Remy-Jardin M, Remy J, Wallaert B, Müller NL. Subacute and chronic bird breeder hypersensitivity pneumonitis: Sequential evaluation with CT and correlation with lung function tests and bronchoalveolar lavage. Radiology 1993;189:111-8.

7. Khoor A, Leslie KO, Tazelaar HD, Helmers RA, Colby TV. Diffuse pulmonary disease caused by nontuberculous mycobacteria in immunocompetent people (hot tub lung). Am J Clin Pathol 2001;115:755-62.

8. Yamazaki Y, Kubo K, Sekiguchi M, Honda T. Analysis of BAL fluid in M. avium-intracellulare infection in individuals without predisposing lung disease. Eur Respir J 1998;11:1227-31.

9. Heurlin N, Bergström SE, Andersson J, Christensson M, Christensson B. Lack of T-lymphocytosis and poor interferon gamma production in BAL fluid from HIV-negative immunocompetent patients with pulmonary non-tuberculous mycobacteriosis. Scand J Infect Dis 1998;30:339-43.

10. Corrin B, Nicholson AG. Pathology of the Lungs, 2nd edn. United Kingdom: Churchill Livingstone, 2005:644. 


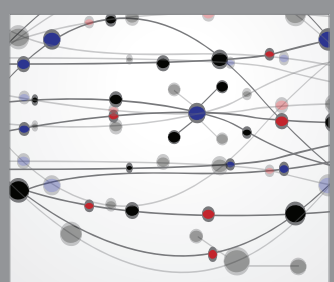

The Scientific World Journal
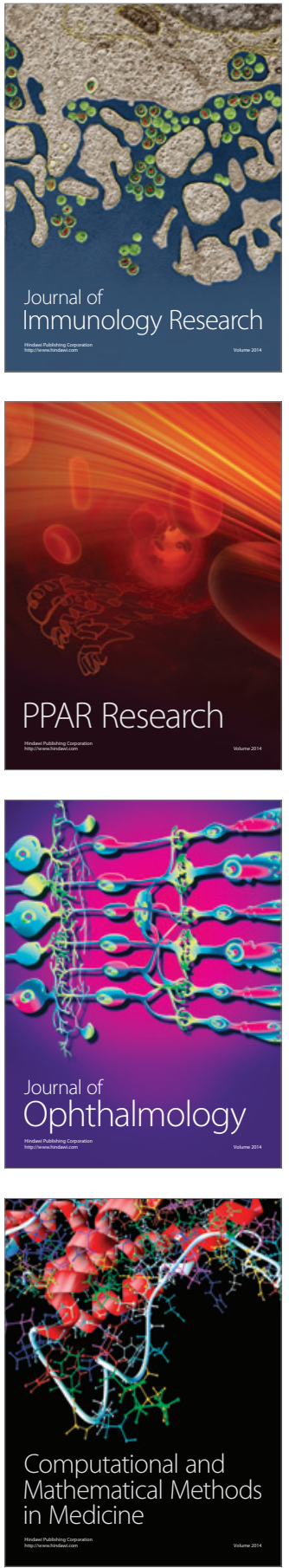

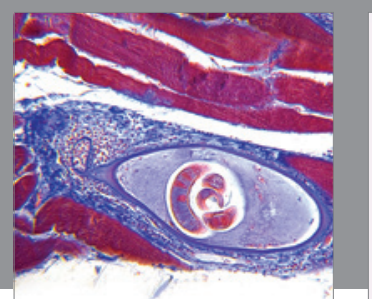

Gastroenterology Research and Practice

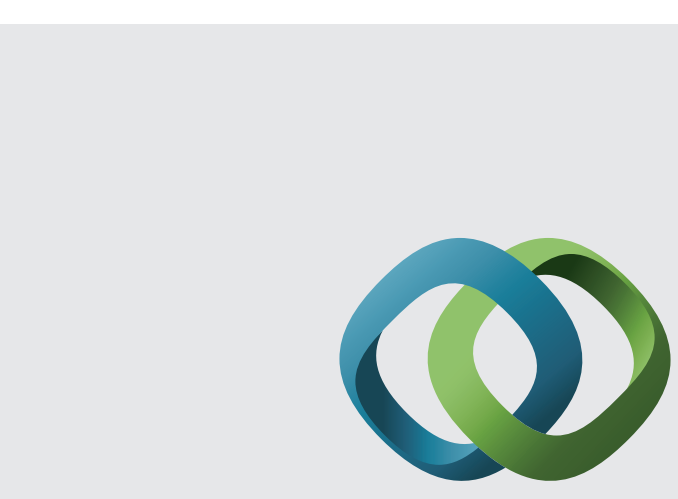

\section{Hindawi}

Submit your manuscripts at

http://www.hindawi.com
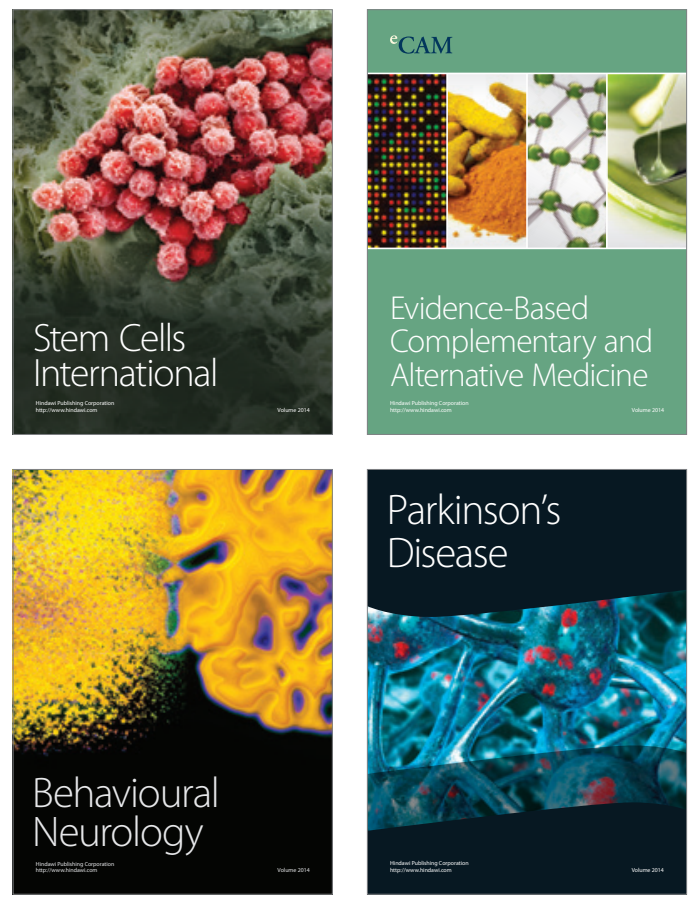
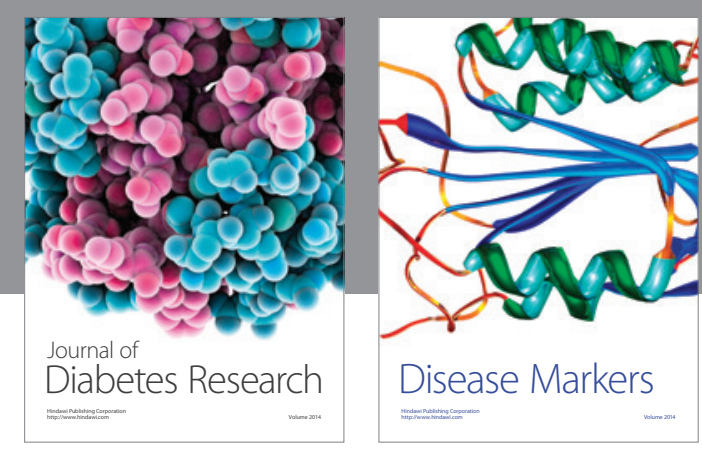

Disease Markers
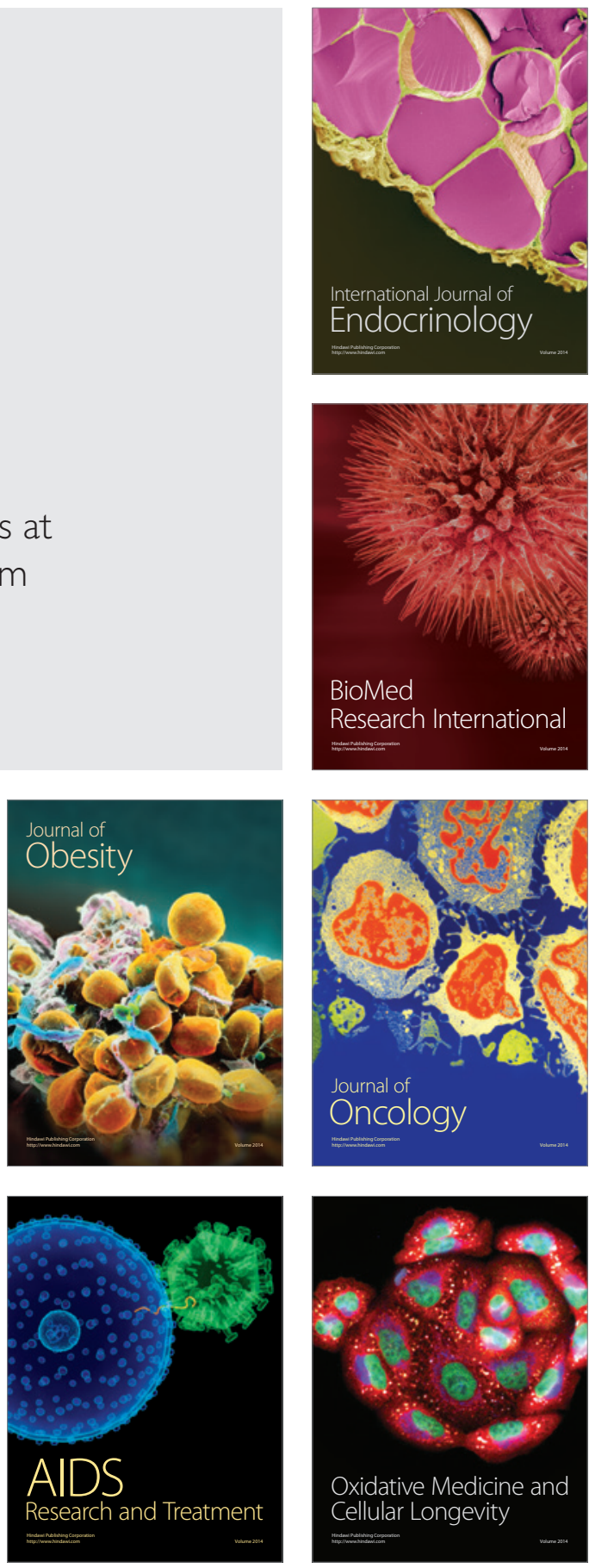